\title{
DINOPHYCEAE DO ESTADO DE MATO GROSSO, MT, BRASIL: MUNICÍPIOS DE BARRA DO BUGRES, CÁCERES, CHAPADA DOS GUIMARÄES, PORTO ESPERIDIÃO E QUATRO MARCOS.'
}

\author{
Mariângela Menezes ${ }^{2}$ \\ Valéria de Oliveira Fernandes ${ }^{3}$
}

Recebido em 20-2-90. Aceito em 22-10-90

RESUMO - São apresentados estudos taxonômicos preliminares das Dinophyceae tecadas, baseados em amostras coletadas em julho de 1983 e novembro de 1984, nos Municípios de Barra do Bugres, Cáceres, Chapada dos Guimarães, Porto Esperidião e Quatro Marcos, Estado de Mato Grosso, Brasil. Foram identificados três taxons do gênero Peridinium Ehr. e um de Sphaerodinium Wol. Todas as espécies constituem primeira citação de ocorrência para o Estado de Mato Grosso e uma (P. africanum Lemm.) para o Brasil.

Palavras-chave: Dinophyceae, Taxonomia, Mato Grosso, Brasil.

ABSTRACT - A preliminary taxonomical survey was carried of the thecate Dinophyceae based on samples collected in July 1983 and November 1984 from Barra do Bugres, Cáceres, Chapada dos Guimarães, Porto Esperidião and Quatro Marcos Municipalities, State of Mato Grosso, Brazil. Three taxa belonging to the genus Peridinium Ehr. and one species of Sphaerodinium Wol. were identified. All species are new records for the Mato Grosso State and one species $(P$. africanum Lemm.) for Brazil.

\section{Introdução}

O presente trabalho visa prosseguir o estudo da Ficoflórula na área sob influência da BR-364 (Cuiabá-Porto Velho), Programa Polonoroeste/CNPq/Museu Nacional-UFRJ, iniciado em 1983, tendo parte de seus resultados publicados por Dias (1986, 1989), Menezes (1986), Menezes \& Fernandes $(1987,1989)$ e Sophia \& Silva (1989). Este estudo constitui contribuição pioneira ao conhecimento dos Dinoflagelados (Dinophyceae) para o Estado de

1 - Parte do subprojeto "Estudos botânicos na área sob influência da BR-364 (Cuiabá-Porto Velho)": Programa Polonoroeste, CNPq/Museu Nacional-UFRJ.

2 - Departamento de Botânica, Museu Nacional, Quinta da Boa Vista, São Cristóvão, 20942 - Rio de Janeiro, RJ. Bolsista do CNPq.

3 - Departamento de Ecologia, Instituto de Biologia, Cidade Universitária, Ilha do Fundão, 21941 - Rio de Janeiro, RJ. Bolsista do CNPq. 
Mato Grosso e está baseado em amostras da I e II Expedições do Programa a cinco Municípios do Estado.

\section{Material e Métodos}

As amostras foram coletadas nos Municípios de Barra do Bugres, Cáceres, Chapada dos Guimarães, Porto Esperidião e Quatro Marcòs, MT, Brasil (Figura 1), nos meses de julho/83 e novembro/84.

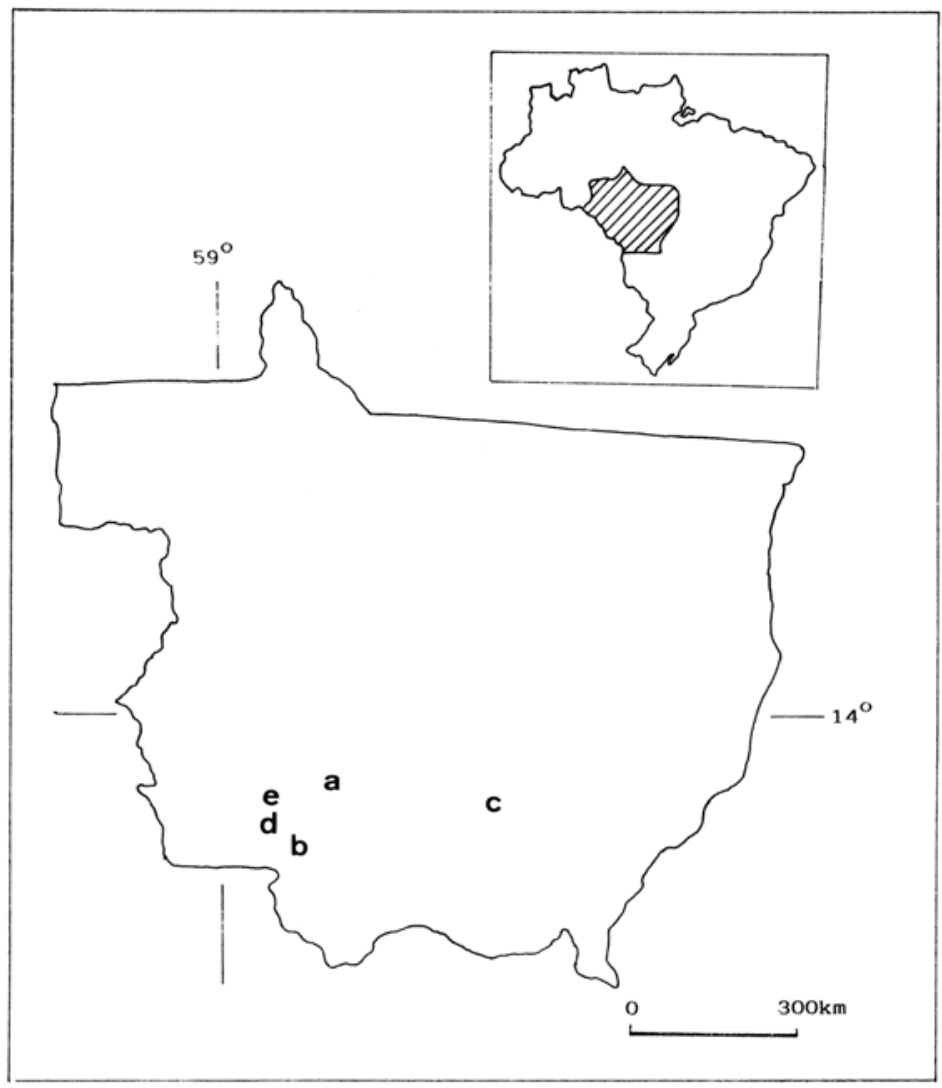

Fig. 1 - Mapa do Estado de Mato Grosso indicando os Municípios de coletas: a - Barra do Bugres; b - Cáceres; c - Chapada dos Guimarães; d - Porto esperedião; e - Quatro Marcos. 
As coletas foram efetuadas utilizando-se a seguinte metodologia: passagem de rede de plâncton de $25 \mu \mathrm{m}$ de abertura de malha; espremido manual de partes submersas de vegetação aquática; passagem de frasco na superfície da água e recolhimento de sedimento. Todo o material foi fixado em solução de Transeau e incluído no Herbário do Museu Nacional do Rio de Janeiro (R).

Como todas as amostras foram analisadas fixadas, só foram identificados os indivíduos tecados com lóricas íntegras. Para evidenciar as placas das tecas das Dinophyceae, procedeu-se à diafanização do material com hipoclorito de sódio a $50 \%$ e posterior coloração com cloreto de zinco iodado (Lefêvre, 1932). O sistema de tabulação utilizado seguiu Kofoid (1909) e a identificação dos táxons baseou-se em Huber-Pestalozzi (1950), Popovský \& Pfiester (1986), Schiller (1937), Starmach (1974) e Thompson (1950).

Do total das 233 amostras examinadas somente as sete discriminadas abaixo apresentaram representantes de Dinophyceae:

- Município de Chapada dos Guimarães, Rio Manso, Salto da Praia Rica, remanso, fitoplâncton, col. V. L. Huyszar e M. Menezes, s/n, 21.XI.84 (R149611).

- Município de Porto Esperidião, Fazenda Pantanalzinho, Charco do Rio Aguapeí, pelon, col. V. L. Huszar, s/n, 24.XI.84 (R155574).

- Município de Quatro Marcos, Fazenda Favorita, Açude da Fazenda, metafíton, col. V. L. Huszar, s/n, 29.XI.84 (R155581).

- Município de Quatro Marcos, Fazenda Favorita, Açude da Fazenda, curral, fitoplâncton, col. V. L. Huszar, s/n, 29.XI.84 (R155582).

- Município de Cáceres, BR-174 (Cáceres-Pontes e Lacerda), km 45, alagado do Córrego Caramujo, fitoplâncton, col. V.L. Huszar, s/n, 01.XII.84 (R155585).

- Município de Barra do Bugres, Fazenda Bocaina, Açude da Fazenda, metafíton, col. V.L. Huszar, s/n, 04.XII.84 (R155589).

- Município de Porto Esperidião, Fazenda Pantanalzinho, perifíton, col. V.L. Huszar, s/n, 24.XI.84 (R169669).

\section{Resultados e Discussão}

A análise das amostras permitiu a identificação de quatro taxa, todos constituindo os primeiros registros para a área estudada e um primeiro para o Brasil. 


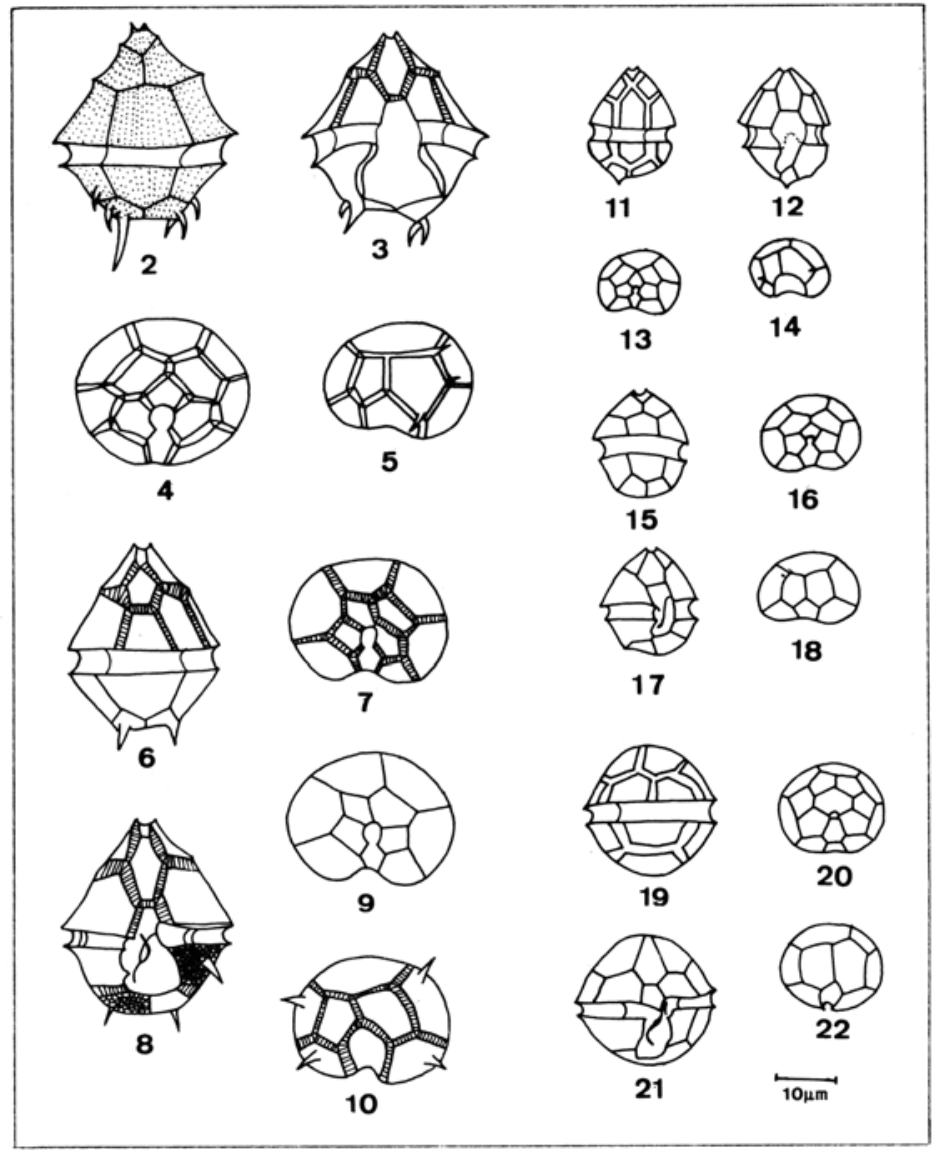

Figura 2-3 - Peridinium africanum Lemm.

Figura 6-10 - Peridinium cunningtonii (Lemm.) Lemm.: 7 - tab. remotum Lef.; 9 tab. contactum Lef.

Figura 11-18 - Peridinium umbonatum Stein var. umbonatum: 11,13 - tab. contactum (Lef.) Lef.; 15,16 - tab. remotum Lef.

Figura 19-22 - Sphaerodinium cinctum (Ehr.) Wol. 
Peridinium africanum Lemm. Jour. Linn. Soc. Bot. 38: 188. 1907. Figuras 2-5

Células pentagonais, não achatadas dorsiventralmente; epicono cônico e maior que o hipocono; hipocono truncado a levemente arredondado; cíngulo equatorial, circunscrevendo as células com 6 placas; sulco extendendo-se levemente no epicono, alargando-se no hipocono, atingindo o pólo antapical; poro apical; placas planas ou côncavas, finamente pontuadas em linhas longitudinais; número variado de pequenos dentes ou espinhos na margem do hipocono ou nas placas antapicais, às vezes com espinhos longos e robustos, $5,0-10,0 \mu \mathrm{m}$ compr.; suturas retas, estriadas.

Dimensões: $30,0-41,4 \mu \mathrm{m} \times$ 23,0-30 um.

Tabulação: 4' + 2a + 7"; 5" ' + 2" "'.

Distribuição geográfica no Brasil: primeira citação de ocorrência.

Material examinado: R169669.

Peridinium africanum ocorreu em uma única amostra juntamente $\operatorname{com} P$. umbonatum Stein. Apesar de incluídas na Secção Umbonatum, as duas espécies podem ser separadas pelo hipnozigoto oval a esférico e cíngulo espiralado em $P$. umbonatum, enquanto $P$. africanum apresenta-os cordiforme e equatorial, (Popovský \& Pfiester, 1986).

Nas populações examinadas de ambas as espécies, não foi identificado nenhum estádio de resistência e, portanto as mesmas foram separadas somente com base no cíngulo. Ainda, não se observou qualquer variação morfológica na disposição das placas $P$. africanum, que mostrou sempre tabulação remotum Lef.

Peridinium cunningtonii (Lemm.) Lemm. KryptogFlora Brandenburg. 671,fig. 29-33. 1910.

Basiônimo: Peridiniopsis cunningtonii Lemm., Jour. Linn. Soc. Bot., 38: 189, pl. 9, fig. 2a-c. 1907.

Figuras 6-10

Células pentagonais, não achatadas dorsiventralmente; epicono cônico, maior que o hipocono; hipocono arredondado a levemente truncado; cíngulo espiralado para a esquerda, com 6 placas; sulco extendendo-se no epicono, alargando-se no hipocono, não atingindo o pólo antapical; poro apical; placas côncavas ou levemente convexas, reticuladas; placas antapicais e pós-cingulares p1"' e p5" com espinhos longos e robustos, até ca. 8,0 $\mu \mathrm{m}$ compr.; suturas retas, estriadas.

Dimensões: $32,0-48,0 \mu \mathrm{m} \times 21,3-30,0 \mu \mathrm{m}$

Tabulação: 4' + 1a + 6"; 5" ' + 2"' '. 
Distribuição geográfica no Brasil: AM: Rio Negro, Rio Branco, Rio Cueiras (fitoplâncton) (Uherkovich \& Rai, 1979); MT: primeira citação de ocorrência.

Material analisado: R155581; R155582; R155589.

A circunscrição desta espécie é extremamente confusa, uma vez que os caracteres aplicados na separação dos gêneros Glenodinium, Peridinium p.p. e Peridiniopsis p.p. não se apresentam bem definidos. Glenodinium, particularmente, é o gênero mais controvertido, cuja principal característica se resume à presença de uma membrana muito fina, aparentemente sem tabulação, ao contrário de Peridinium e Peridiniopsis, com tecas verdadeiras (Bourrelly, 1970). No entanto, alguns taxa com nítida tabulação, são incluídos em Glenodinium, tal como G. oculatum Stein (3' + 1a + 7'; 5" , + 2", '). Quanto a Peridinium e Peridiniopsis, estes diferem entre si pela tabulação do epicono: 4' + 2-3a + 7' ' no primeiro e 4' + 0-1a + 6' no segundo.

Huber-Pestalozzi (1950) reconheceu o gênero Glenodinium e incluiu todas as espécies de Peridiniopsis em Peridinium, ao contrário de Bourrelly (1968, 1970), que considerou Peridiniopsis com todas as suas espécies descritas por Lemmermann (1904), além de englobar nesse gênero as espécies de Glenodinum auct. Schiller.

Em sua abordagem sobre os "Problemas na determinação de dinoflagelados de água doce" Popovský (1983) indicou diferentes relações na posição e morfologia das placas entre algumas espécies de Peridiniopsis e entre este e Peridinium. Para o autor, é possível que exista somente uma espécie de $\mathrm{Pe}$ ridiniopsis, $P$. quadridens (Stein) Bourr., com uma grande variabilidade morfológica externa. Concluiu, então, que somente o uso da morfologia externa não é suficiente para a determinação de muitas espécies de Dinoflagelados, desde que os mesmos ocupam na natureza, um nível de organização monado, com diferentes manifestações morfológicas e fisiológicas.

Boltovskoy (comunicação pessoal) acredita que o gênero Peridiniopsis está formado por duas espécies bem definidas: $P$. borgei Lemm.e $P$. cristatum (Bal.) Bourr. e que suas demais espécies, assim como aquelas de Glenodinium, devem ser redistribuídas em outros gêneros existentes, ou até mesmo em novos gêneros. $\mathrm{O}$ mesmo autor sugere ainda, estudo de revisão nos dinoflagelados de água doce baseado no número de placas cingulares, tais como os marinhos. Dessa forma, até que se esclareça a posição taxonômica de Peridiniopsis, os exemplares coletados em Mato Grosso foram identificados como Peridinium cunningtonii seguindo Huber-Pestalozzi (1950).

Os indivíduos analisados mostraram dois tipos de tabulação: remotum (Lind.) Lef. (Figura 7) e contactum Lef. (Figura 9), esta última mais rara, aparecendo aproximadamente em $18 \%$ do total de 45 exemplares examinados. 
Peridinium umbonatum Stein var. umbonatum. Organ. Infus. 3(2), pl. 12, fig. 1-8, 1883 .

Figuras 11-18

Células ovadas e pentagonais, achatadas dorsiventralmente; epicono cônico, maior que hipocono; hipocono arredondado a truncado; cíngulo espiralado para a esquerda, com 6 placas; sulco extendendo-se levemente no epicono, alargando-se no hipocono, nãó atingindo o pólo antapical; poro apical; placas côncavas ou convexas, lisas, às vezes com finas papilas em filas longitudinais, raramente pontuadas, podendo apresentar um dente ou espinhos no lado posterior; suturas retas, lisas, algumas vezes com grânulos entre as placas antapicais.

Dimensões: $21,0-22,0 \mu \mathrm{m} \times 16,0-21,0 \mu \mathrm{m}$

Tabulação: 4' + 2a + 7"; 5"' + 2" "'.

Distribuição geográfica no Brasil: MG; Parque Nacional de Itatiaia, Brejo da Lapa (identificação duvidosa) (Bicudo \& Ventrice, 1968); Parque Nacional de Itatiaia, Lagoa das Prateleiras (Bicudo \& Bicudo, 1969); AM: Rio Branco (Uherkovich \& Rai, 1979: Uherkovich \& Franken, 1980, como $P$. inconspicuum);

Rio Maués (Uherkovich, 1981, como P. inconspicuum); MT: primeira citação de ocorrência.

Material examinado: R149611; R155579; R155589; R169669.

Segundo Popovský \& Pfiester (1986) P. umbonatum, assim como as demais espécies incluídas na Secção Umbonatum, apresentam a mesma tabulação, ocorrendo em condições ecológicas similares, o que as tornam muito próximas entre si. Os mesmos autores ao estudarem algumas espécies desta Secção, indicaram $P$. umbonatum e $P$. inconspicuum Lemm. como cosmopolitas, com morfologia altamente variável, apresentando-se de difícil, às vezes impossível separação, uma vez que diferem entre si somente pelas dimensões de comprimento: $25,0-40,0 \mu \mathrm{m}$ e $15,0-30,0 \mu \mathrm{m}$, respectivamente. Baseados em populações naturais e em bibliografia, Popovsḱ́ \& Pfiester (1986) consideraram $P$. inconspicuum como sinônimo de $P$. umbonatum, desde que os aspectos morfológicos e morfométricos diacríticos são comuns a ambos os taxa, ocorrendo intergradação de uma espécie à outra. Concorda-se com os referidos autores de que as duas espécies representam uma única e os exemplares examinados foram identificados $\operatorname{com} P$. umbonatum var. umbonatum.

Os indivíduos observados apresentaram variação morfológica na disposição das placas, exibindo tabulação remotum Lef. (Figura 16) e tabulação contactum (Lef.) Lef. (Figura 13), esta última aparecendo em $75 \%$ no total de 65 exemplares examinados. Peridinium umbonatum var. umbonatum foi o táxon mais representativo no material estudado, ocorrendo em quatro das sete amostras analisadas. 
Sphaerodinium cinctum (Ehr.) Wol. Bull. int. Acad. Sci. Lett. Cracovie, sér. B, 1/3: 116. 1917.

Basiônimo: Glenodinium cinctum Ehr. Infus. 257, pl. 22, fig. 22. 1838.

Figura 19-22

Células subesféricas, levemente achatadas dorsiventralmente; epicono maior que o hipocono; cíngulo espiralado para a esquerda, com 5 (?) placas; sulco extendendo-se ligeiramente no epicono, alargando-se no hipocono, sem atingir o pólo antapical; poro apical; placas pentagonais planas ou côncavas, lisas, raramente com finas aréolas; placas intercalares aproximadamente isodiamétricas, terceira placa apical hexagonal; suturas retas, lisas, raramente com papilas entre as placas antapicais.

Dimensões: 32,2-37,0 $\mu \mathrm{m}$ X 30,0-35,0 $\mu \mathrm{m}$.

Tabulação: 4' + 4a + 7"'; 6", + 2", ,

Distribuição geográfica no Brasil: RJ: Manguinhos (Prowazek, 1910 como $G$. cinctum); MT: primeira citação de ocorrência.

Material examinado: R169669.

Sphaerodinium cinctum foi proposto por Woloszynska (1917) a partir de Glenodinium cinctum Ehr., espécie-tipo deste último gênero.

Bourrelly (1968) chamou a atenção da impossibilidade da mudança efetuada por Woloszynska, pois segundo o Código de Nomenclatura Botânica (Art. 69), não se pode transferir a espécie-tipo de um gênero para o nome de outro gênero. Acrescentando que as descrições de Ehrenberg (1838) para $G$. cinctum eram extremamente sumárias, Bourrelly (1968) reconheceu o gênero Sphaerodinium, da mesma forma que Lindemann (1928) e Schiller (1937), que consideraram $G$. cinctum como sinônimo de $S$. cinctum. Leoblich (1980) fez um estudo da validade de ambos os taxa e considerou o gênero Sphaerodinium como sinônimo de Glenodinium, desde que todas as espécies de Sphaerodinium propostas por Woloszynska apresentam a mesma configuração do estigma que $G$. cinctum, isto é, em forma de ferradura. Porém, em decorrência do processo de fixação, esta característica não foi observada nos exemplares mato-grossenses, sendo a determinação taxonômica dos mesmos baseada na morfologia das tecas. Como as descrições e ilustrações de Ehrenberg (1838) não indicam a tabulação de $G$. cinctum, optou-se por incluir os exemplares estudados no gênero Sphaerodinium, identificando-os com $S$. cinctum. Os indivíduos analisados mostraram sempre as quatro placas apicais aproximadamente isodiamétricas e a terceira placa apical hexagonal, o que segundo Thompson (1950) é típico do gênero Sphaerodinium.

A maioria das células mostrou estruturas pouco visíveis e nenhuma delas apresentou reação positiva na presença do corante. As tecas apresentaram-se hialinas, frágeis, de fácil deformação, discordando em parte da descrição de Huber-Pestalozzi (1950), que referiu as células de $S$. cinctum como resistentes e de membrana firme. 
Dinophyceae do Estado de Mato Grosso...

\section{Agradecimentos}

Ao Dr. Andrés Boltowskoy, Universidade de la Plata, Argentina, pelas informações cedidas e à Profa. Lúcia Helena Sampaio da Silva, pela revisão do texto.

\section{Referências Bibliográficas}

BICUDO, C.E.M \& R.M. BICUDO. 1969. Algas da Lagoa das Prateleiras, Parque Nacional do Itatiaia, Brasil. Rickia 4:1-39.

BICUDO, C.E.M \& M.R. VENTRICE. 1968. Algas do Brejo da Lapa, Parque Nacional do Itatiaia, Brasil. Anais XIX Congr. Soc. Bot. Bras. p.1-30.

BOURRELLY, P. 1968. Notes sur les Péridiniens d'eau douce. Protistologica 4(1):5-14.

BOURRELLY, P. 1970. Les algues d'eau douce: iniciation à la systématique 3: les algues bleues et rouges, les Eugleniens, Péridiniens et Cryptomanadines. Editions N. Boubée \& Cie.

DIAS, I.C.A. 1986. Resultados preliminares sobre a ficoflórula da Chapada dos Guimarães e arredores, Mato Grosso, Brasil: Zygnemaceae (Zygnemaphyceae). Rickia 13:69-75.

DIAS, I.C.A. 1989. Chlorophyta filamentosas do Município de Cáceres e arredores, Mato Grosso, Brasil. Acta Bot. Bras. 3(2): 3-12 EHRENBERG, C.G. 1838. Die Infusionsthierchen als vollkommene Organismen: ein Blick in das Tiefere organische Leben der Natur. Verlag von Leopold Voss, Leipzig.

HUBER-PESTALOZZI, G. 1950. Die Binnengewässer 16. Das phytoplankton des Susswassers 3. Cryptophyceen, Chloronionadinen, Peridineen. E. Schweizerbartsche Verlag Sbuchandlung, Stuttgart.

KOFOID, C.A. 1909. On Peridinium steinii Jorg., with a note on the nomenclature of the skeleton of the Peridiniales. Arch. Protistenk. 16:25-47.

LEFÈVRE, M. 1932. Monographie des espèces d'eau douce du genre Peridinium. Ark. Bot. 2:1-208.

LEMMERMANN, E. 1904. Das plankton schwedischer gewässei. Ark. Bot. 2(2):1-209.

LOEBLICH, A.R. 1980. Dinoflagellate nomenclature. Taxon 29:321-323.

LINDEMANN, E. 1928. Peridineae (Dinoflagellatae). A. In: ENGLER \& K. PRANTL (eds.) Die Naturlichen Planzenfamilien. Vol 2. Wilhelm Engelmann, Lepzig.

MENEZES, M. 1986. Ficoflórula da Chapada dos Guimarães e arredores, Mato Grosso, Brasil: Euglenaceae pigmentadas (Euglenophyceae). Rickia 13:87-95. 
MENEZES, M \& V.O. FERNANDES. 1987. Euglenaceae pigmentadas do Município de Cáceres e arredores, Mato Grosso, Brasil. Rickia 14:53-71.

MENEZES, M \& V.O. FERNANDES. 1989. Euglenaceae (Euglenophyceae) pigmentadas no Noroeste do Estado de Mato Grosso, Brasil: Municípios de Barra do Burges, Cáceres, Juína e Porto Esperidião. Hoehnea 16:35-55.

POPOVSK'Y, J. 1983. Problems in the determination of freshwater dinoflagellates (Dinophyceae). Schweiz. Z. Hydrol. 45(2):365-372.

POPOVSKY', J. \& L.A. PFIESTER. 1986. A taxonomical note to the Section umbonatum of the genus Peridinium Ehrenberg, 1932 (Dinophyceae). Arch. Protistenk. 132:73-77.

PROWAZEK, S. 1910. Contribuição para o conhecimento da fauna de protozoários do Brasil. Mems. Inst. Oswaldo Cruz 2(2):149-158.

SCHILLER, J. 1937. Dinoflagelatae (Peridineae). In: RABENHORST'S KryptogFlora 10(2):1-390.

SOPHIA, M.G. \& L.H.S.SILVA. 1989. Considerações sobre a Flora de Desmidias filamentosas (Zygnemaphyceae) do Noroeste de Mato Grosso e Sudeste de Rondônia, Brasil. Rev. Bras. Biol. 49(4) (no prelo).

STARMACH, K. (ed.) 1974. Cryptophyceae, Dinophyceae, Rabhidophyceae. In: STARMACH, K. (eds) Flora Stodkowodna Polski 4. Warszawa Polska Akademia Nauk Instytut Botaniki. 644p.

THOMPSON, R.H. 1950. A new genus and new records of fresh water Pyrrophyta in the Desmonkontae and Dinophyceae. Lloydia 13(4):277-300.

UHERKOVICH, G. 1981. Algen aus einigen gewässer amazoniens Amazoniana 7(2):191-219.

UHERKOVICH, G. \& M. FRANKEN. 1980. Aufwuchsalgen aus zentralamazonische Pegenwaldebächen. Amazoniana 7(1):49-79.

UHERKOVICH, G. \& H. RAI. 1979. Algen aus dem Rio Negro und seinen Nebenflüssen. Amazoniana 6(4):611-638.

WOLOSZYNSKA, J. 1917. Neue Peridineen-Arten nebst Bemerkungen über deen Bau der Hülle bei gymno und Glenodinium. Bull. Int. Acad. Sci. Lett. Cracovie. sér. B, 1-3:114-120. 